\title{
ExteNDing proteome coverage with legumain as highly specific digestion protease
}

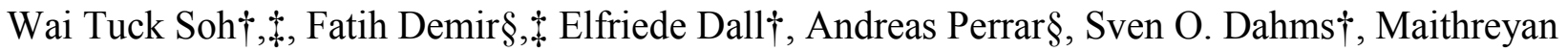
Kuppusamy§, Hans Brandstetter†, Pitter F. Huesgen $\S, \|, \perp, *$

$†$ Department of Biosciences, University of Salzburg, 5020 Salzburg, Austria.

$\S$ Central Institute for Engineering, Electronics and Analytics, ZEA-3, Forschungszentrum Jülich, 52428 Jülich, Germany.

\|Cologne Excellence Cluster on Cellular Stress Responses in Aging Associated Diseases (CECAD), Medical Faculty and University Hospital, University of Cologne, 50931 Cologne, Germany.

$\perp$ Institute for Biochemistry, Faculty of Mathematics and Natural Sciences, University of Cologne, 50674 Cologne, Germany.

† These authors contributed equally.

Corresponding author:

*p.huesgen@fz-juelich.de

\section{Supporting Information:}

\section{Supporting Figures S1-S10.}

Supporting Table S1. Proteins identified after digestion of an Arabidopsis thaliana Col-8 leaf proteome with legumain, GluC or trypsin in three technical replicates per enzyme. (separate xlsspreadsheet)

Supporting Table S2. Label-free quantification of $A$. thaliana Col-8 leaf quantified by at least two peptides after proteome digestion with legumain, GluC or trypsin. (separate xls-spreadsheet)

Supporting Table S3. N-termini identified in the A. thaliana Col-8 leaf proteome using legumain, GluC and trypsin in the HUNTER workflow. (separate xls-spreadsheet)

Supporting Table S4. Identification of N-glycosylation sites in A. thaliana Col-8 leaf apoplast fluid by parallel sequential incubation with PNGase F and legumain and vice versa. (separate $x l s$ spreadsheet) 
(a)

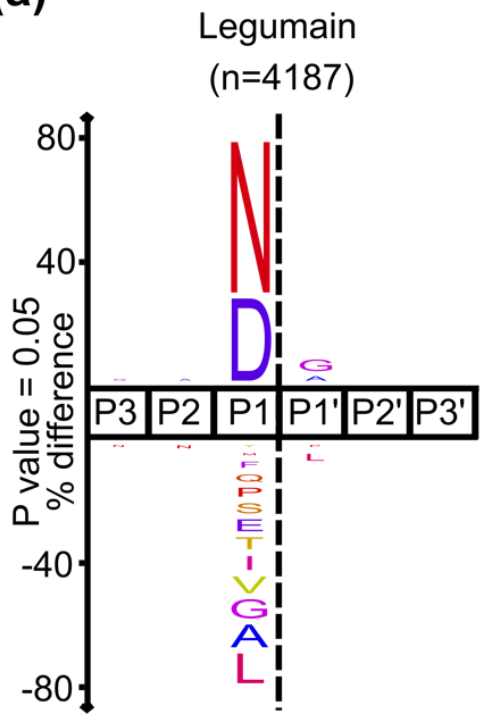

(b)

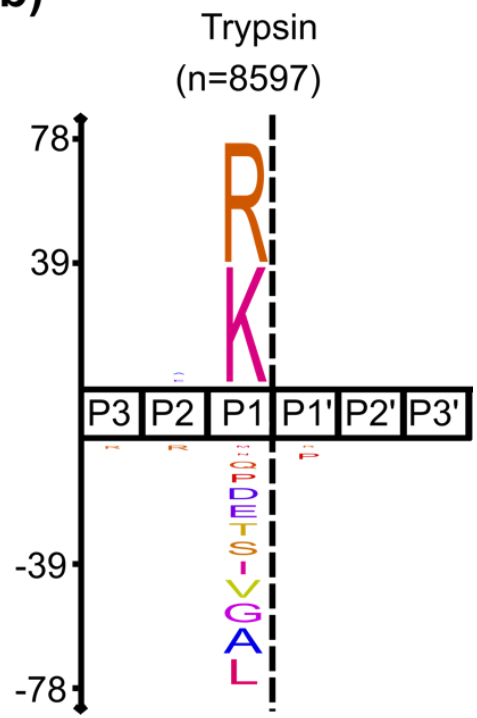

Figure S1. Substrate cleavage specificity of legumain and trypsin in E.coli digests. IceLogos visualize the amino acid frequencies surrounding the cleavage sites inferred from peptides identified by nonspecific database searches after digest of an E.coli proteome with (a) legumain, (b) trypsin. The numbers of non-redundant cleavage sites for each logo are indicated. 
(a)
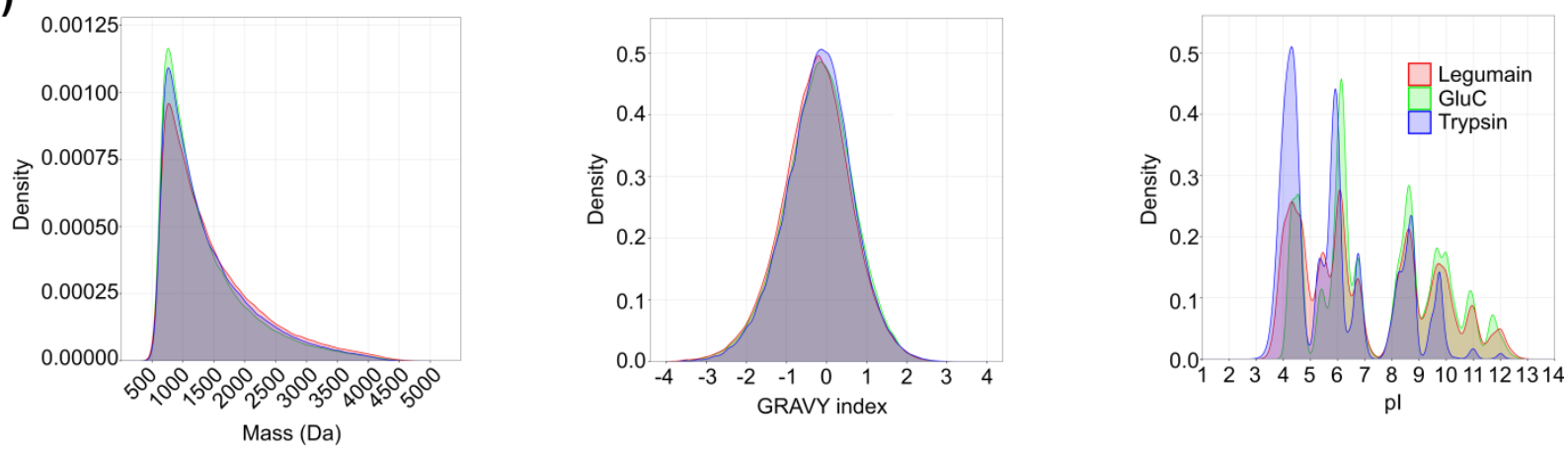

(b)
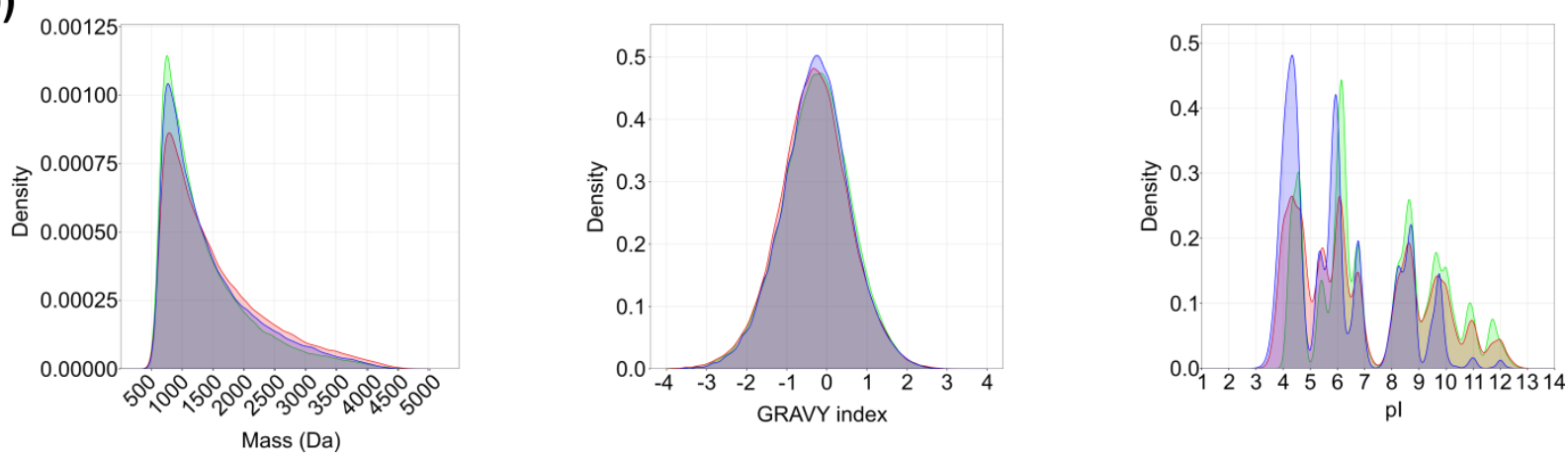

Figure S2. Biophysical properties of peptides derived by in silico digestion of (a) A. thaliana and (b) M. musculus proteomes with the specificity rules for legumain, GluC, and trypsin. Grey area represents overlapping digests. 
(a) SUPEROXIDE DISMUTASE (P24704)/at1g08830

$\begin{array}{lllllll}10 & 20 & 30 & 40 & 50 & 60 & 70\end{array}$

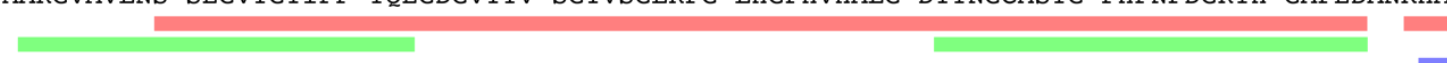

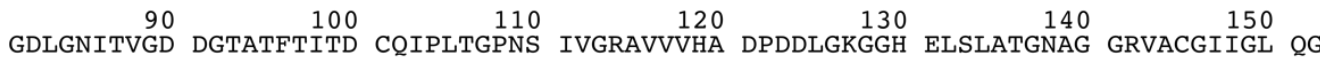

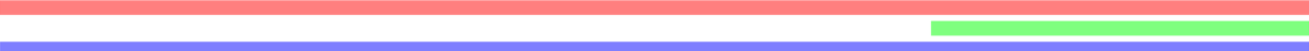

Legumain: $\sim 93 \% \quad$ GluC: $\sim 43 \% \quad \square \quad$ Trypsin: $\sim 49 \%$

(b) GERMIN-IKE PROTEIN 1 (P94040)/at1972610

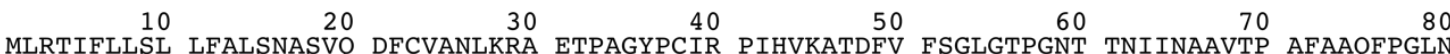

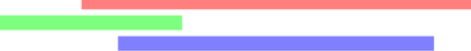

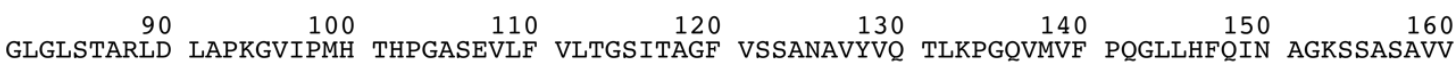

GLGLSTARLD LAPKGVIPMH THPGASEVLF VLTGSITAGE VSSANAVYVQ ILKPGQVMVE PQGLLHEQIN

$\begin{array}{rrrrr}170 & 180 & 190 & 200 & 208 \\ \text { TFNSANPGLQ } & \text { ILDFALFANS } & \text { LPTELVVGTT } & \text { FLDATTVKKL } & \text { KGVLGGTG }\end{array}$

Legumain: $\sim 63 \%$ GluC: $\sim 23 \% \quad$ Trypsin: $\sim 8 \%$

Figure S3. Comparison of protein sequence coverage. Peptides identified in A. thaliana leaf proteome digests with legumain, GluC and trypsin are mapped to the primary sequence of (a) SUPEROXIDE DISMUTASE and (b) GERMIN-LIKE PROTEIN 1. UniProt, AGI accession codes and percentage sequence coverage obtained with each protease are indicated. 
(a)

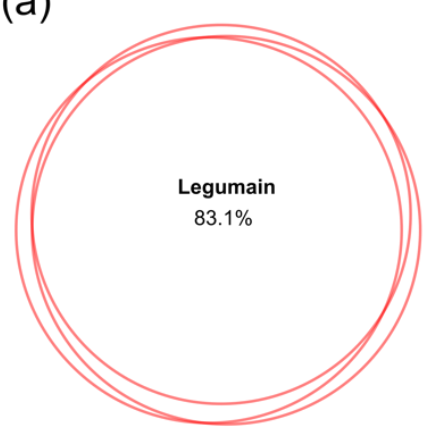

Arabidopsis

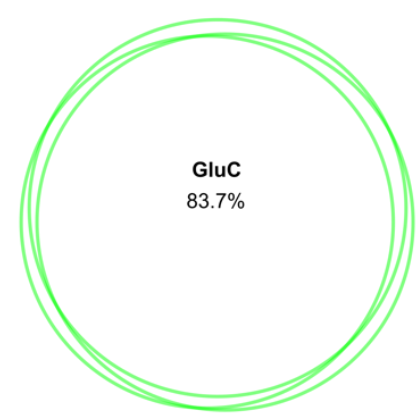

Arabidopsis

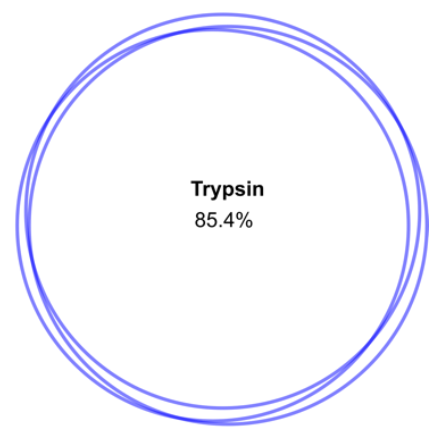

Arabidopsis

(b)

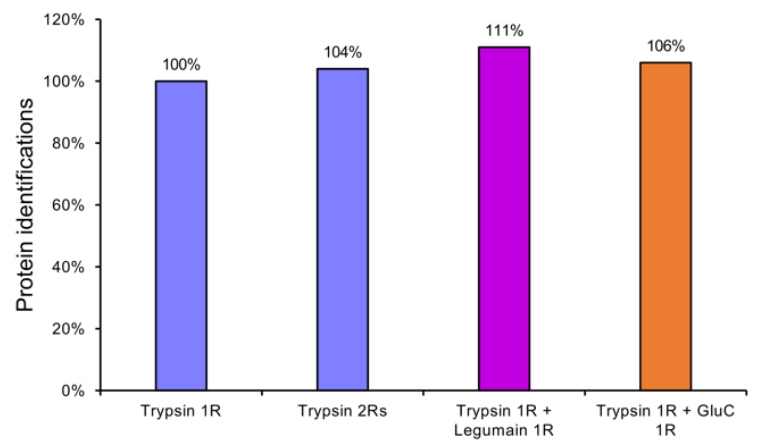

(c)

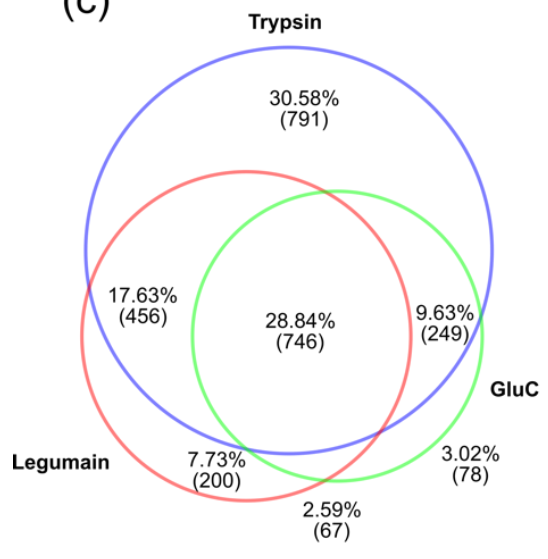

MEF (d)

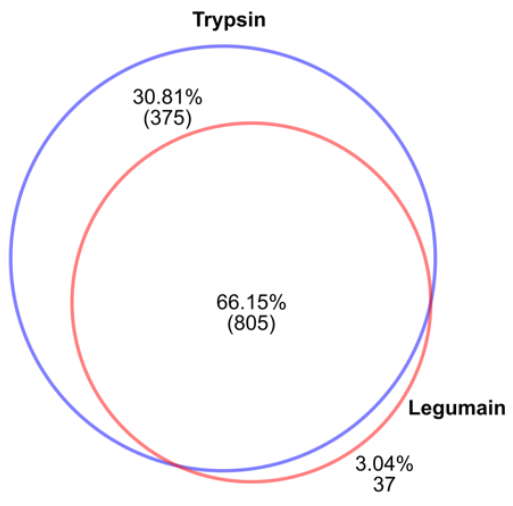

E.coli

Figure S4. Overlap of protein group identifications after digestion with legumain, GluC and trypsin. (a) Overlap in the number of protein groups identified in the three replicate analyses of an $A$. thaliana leaf proteome with each enzyme. (b) Average relative increase in protein group identifications in two tryptic replicate digestions or any combination of a tryptic digestion combined with one legumain or GluC digestion, compared to a single digestion of the A. thaliana proteome with trypsin. (c) Overlap of protein group identifications after digestion of a MEF proteome with legumain, GluC and trypsin. (d) Overlap of protein groups identified in a legumain and a tryptic digest of an E. coli proteome. 

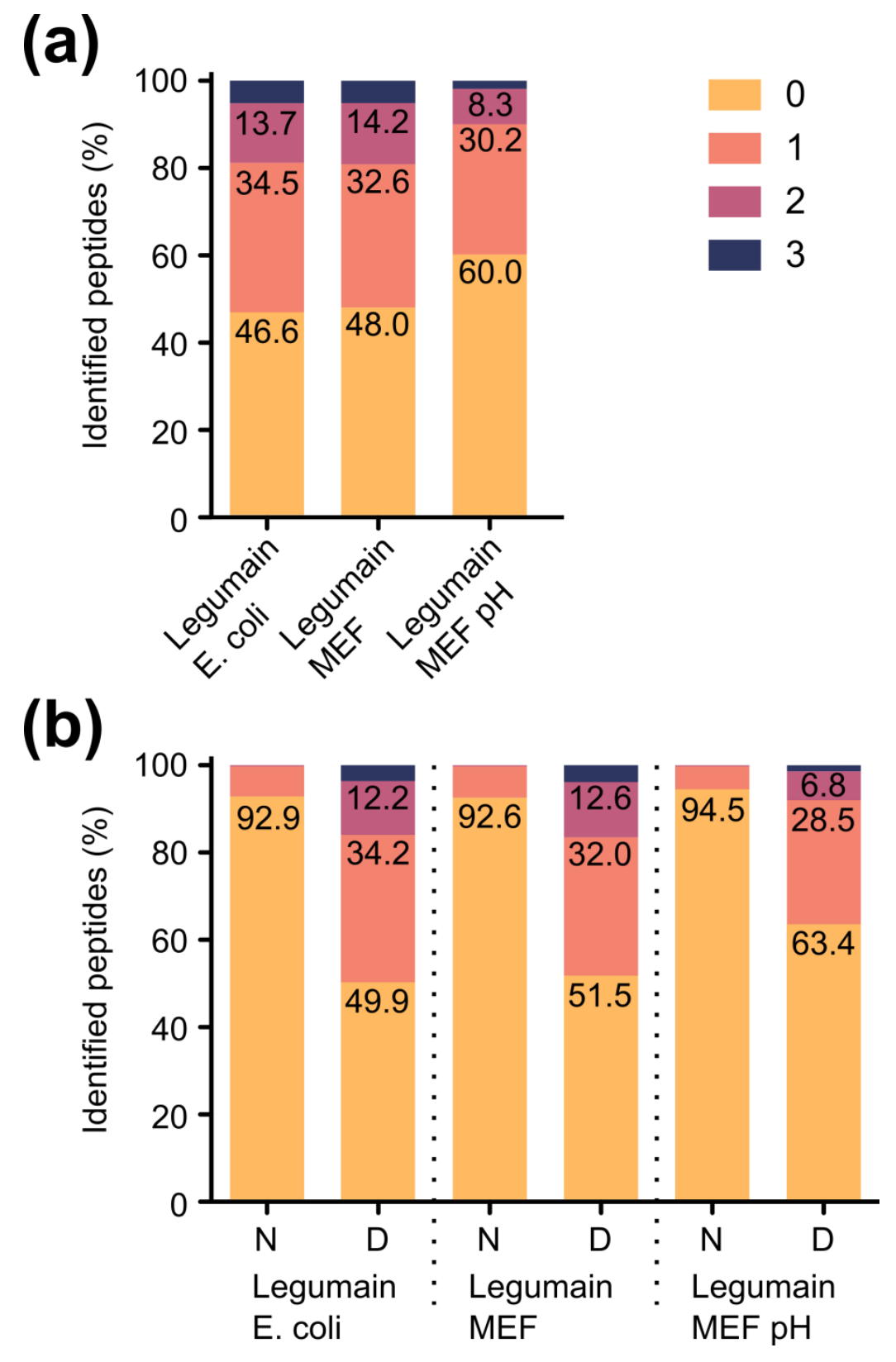

Figure S5. Potential cleavage sites missed by legumain in MEF and E.coli proteome digests at $\mathrm{pH} 6.0$, and in a MEF proteome digest where the $\mathrm{pH}$ was shifted to $\mathrm{pH} 4.0$ after half of the incubation time. (a) total missed cleavages, (b) missed cleavages by amino acid. 


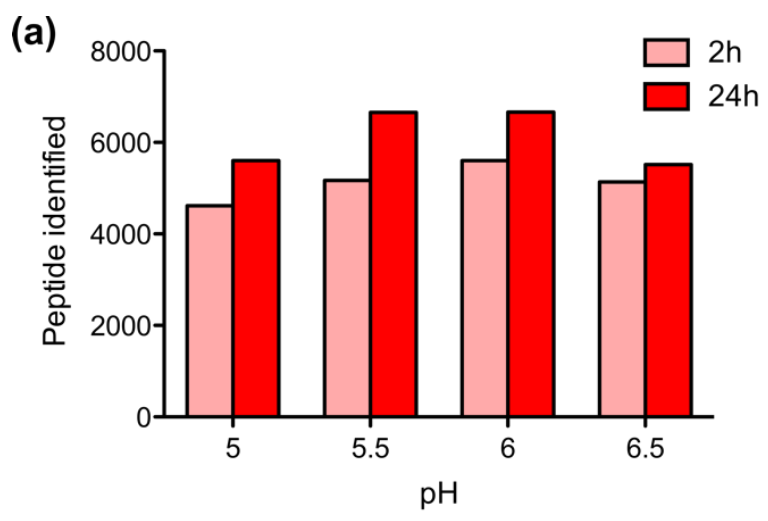

(b)
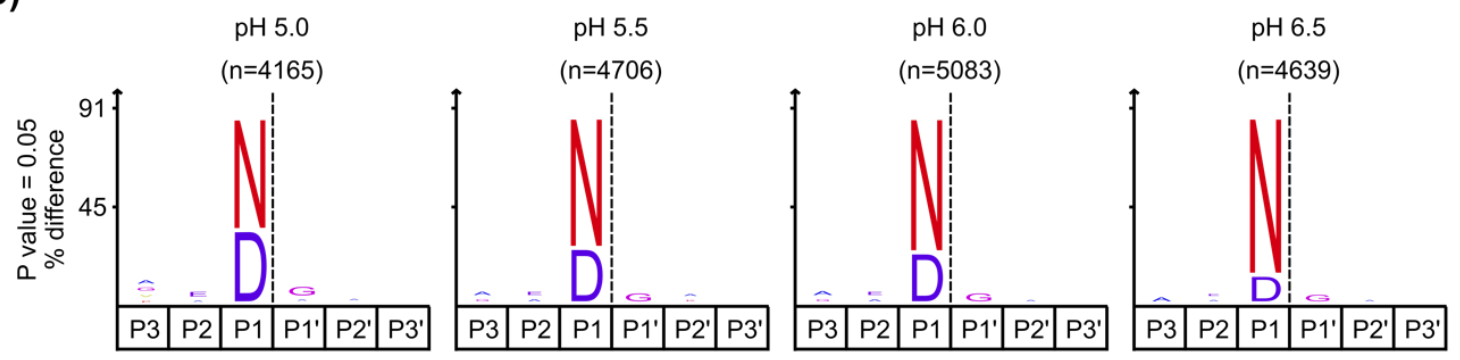

$2 \mathrm{~h}$
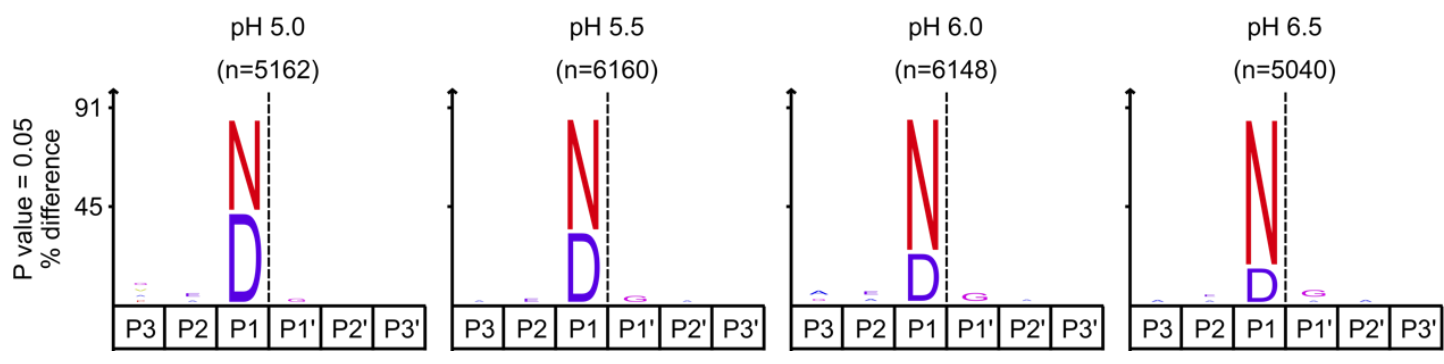

$24 \mathrm{~h}$

Figure S6. Optimization of legumain digestion conditions using A. thaliana proteome. (a) Number of peptides identified in legumain digest incubated at various $\mathrm{pH}$ conditions for 2 and 24 hours. (b) Cleavage specificity analysis of legumain digestion at various $\mathrm{pH}$ conditions for 2 hours and 24 hours visualized by iceLogos based on the identified non-redundant cleavage sites. 
(a)

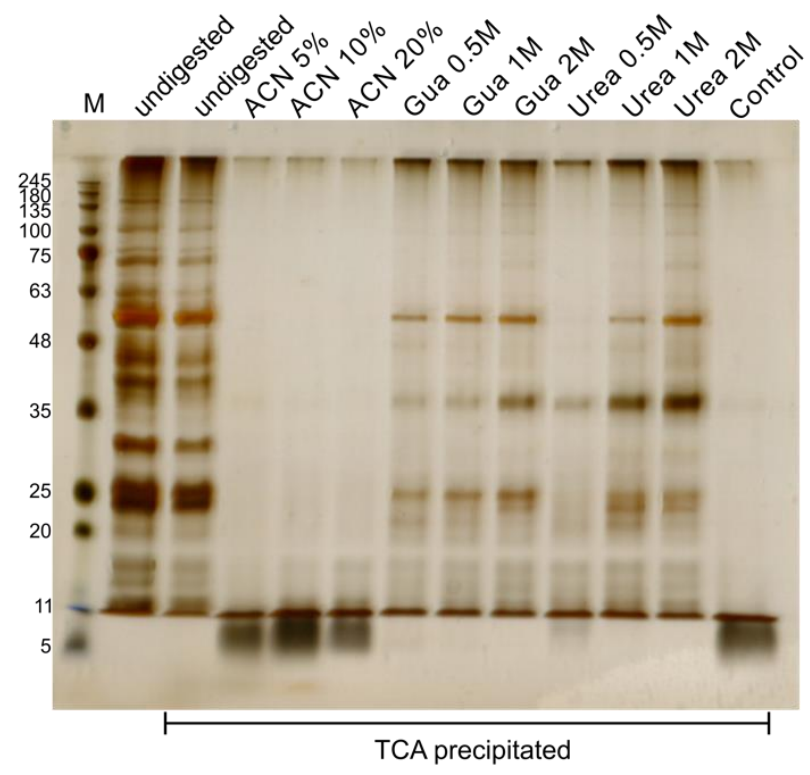

(b)

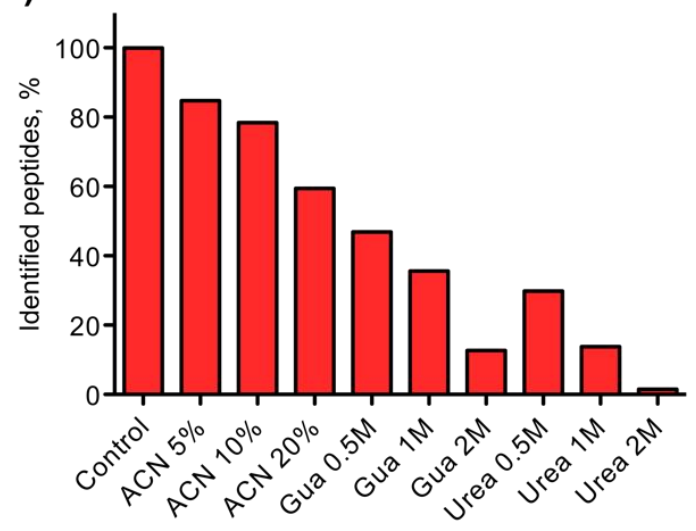

(c)

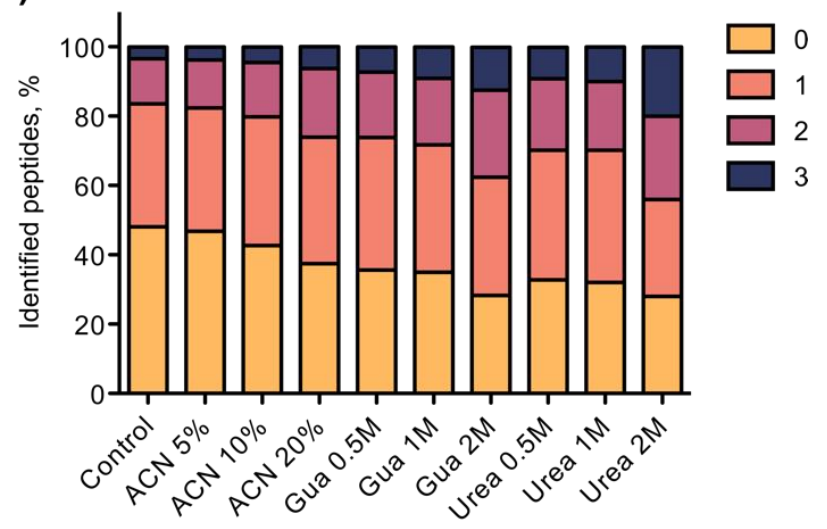

Figure S7. A. thaliana proteome digestion by legumain in the presence of different denaturants used in proteomic experiments. (a) Legumain digestion efficiency in the presence of different denaturants visualized by silver-stained SDS-PAGE. The samples were TCA precipitated to remove the denaturant prior to SDS-PAGE analysis. (b) Number of peptides identified by legumain in the presence of different denaturants at various concentrations. (c) Number of missed cleavage sites in the peptides identified in (b). 

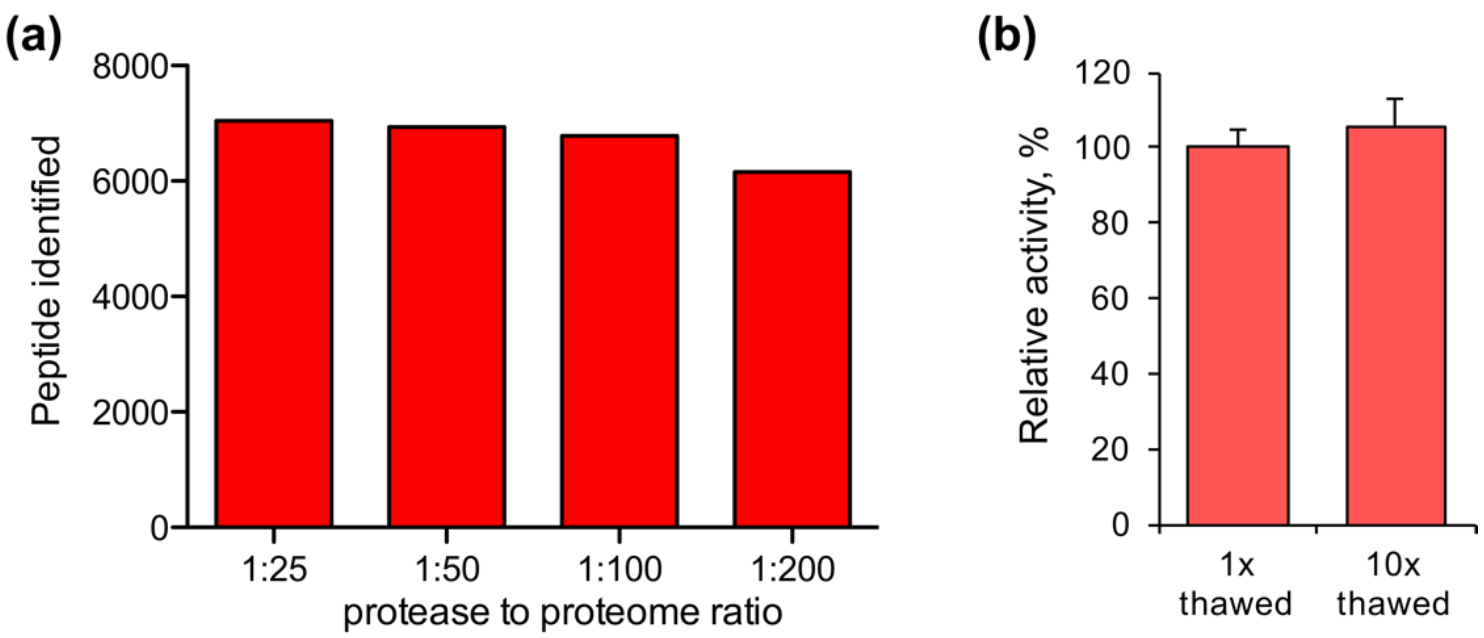

Figure S8. Proteolytic activity of legumain. (a) Peptides identified in legumain digested A.thaliana leaf proteome after incubation at $\mathrm{pH} 6$ at varying protease to proteome ratio. (b) Peptidase activity of legumain after repeated freeze-and-thaw cycles. The experiment was performed using $50 \mu \mathrm{M}$ of AAN-AMC substrate in $50 \mathrm{mM}$ citric acid, $\mathrm{pH} 5.5,100 \mathrm{mM} \mathrm{NaCl}$ at $37{ }^{\circ} \mathrm{C}$ and an enzyme concentration of $8 \mathrm{nM}$. Error bars indicate the standard deviation of the assays $(n=3)$. 


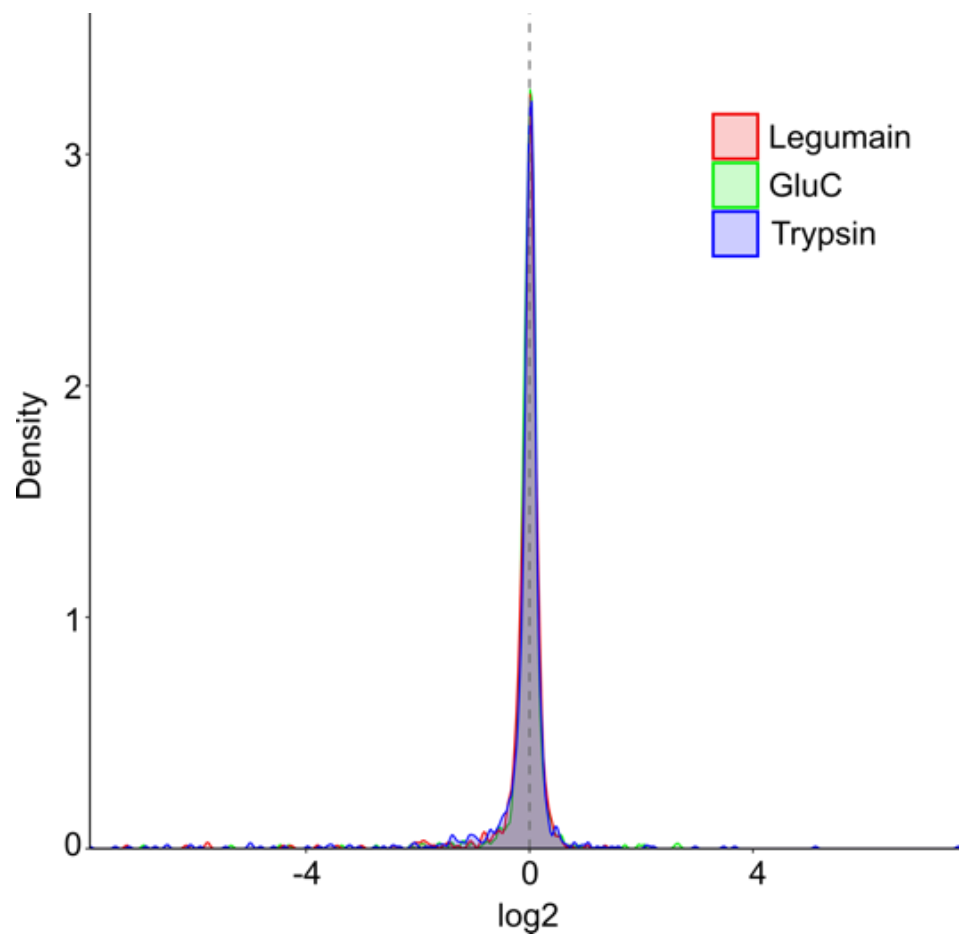

Figure S9. Quantification of protein N-terminal peptides identified in at least two of three replicates of two differentially dimethyl stable isotope labeled aliquots of the same A. thaliana leaf proteome for each enyzme. 
(a)
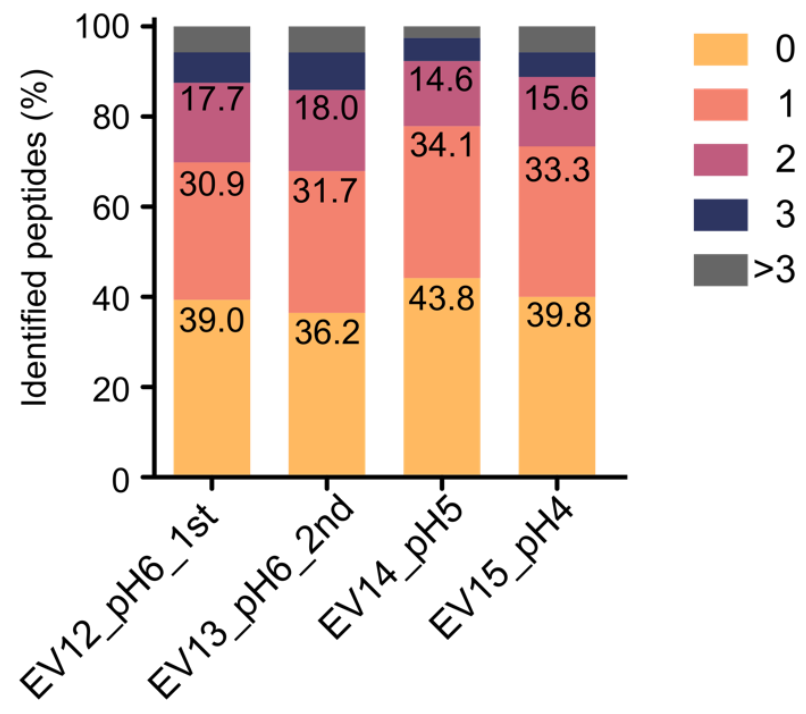

(b)

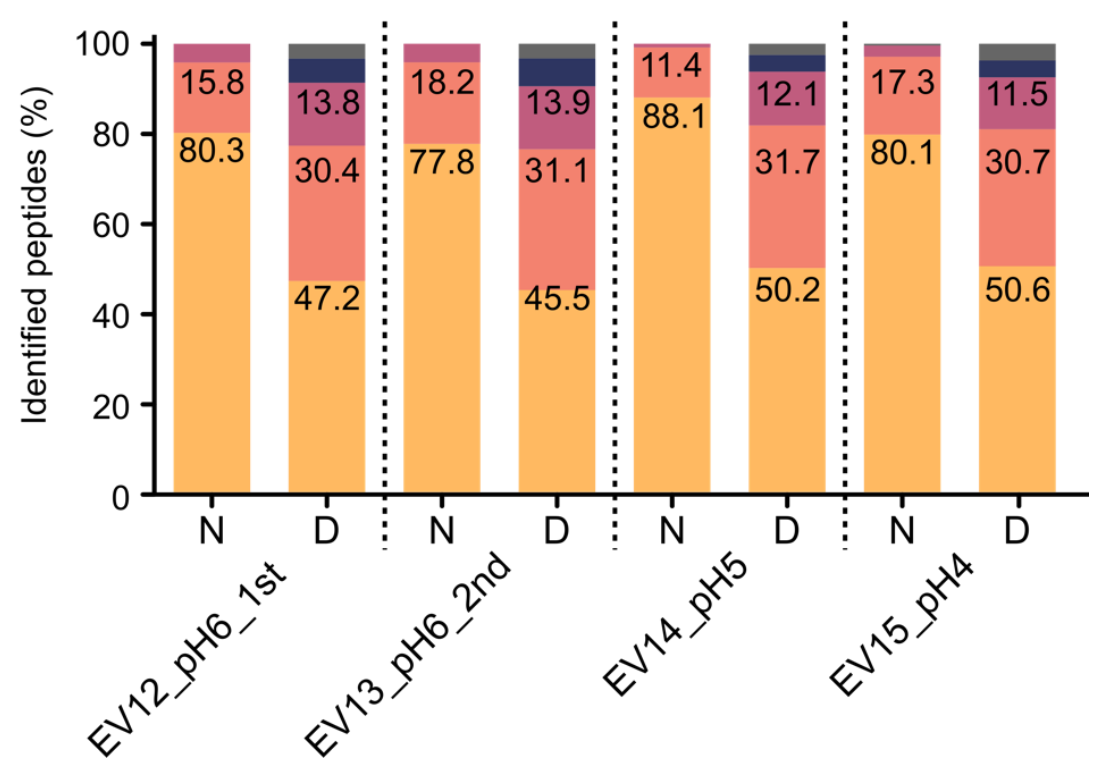

Figure S10. Potential legumain cleavage sites present in peptides identified by Vidmar et al. (2017) after in-gel digest of MDA-MB-231 proteomes at $\mathrm{pH} 4.0, \mathrm{pH} 5.0$ and in two technical replicates at $\mathrm{pH} 6.0$ (a) total missed cleavages, (b) missed cleavages by amino acid. Peptide sequences were retrieved from https://www.embopress.org/doi/10.15252/embj.201796750 